Supporting Information

\title{
Stretchable Transparent Electrode via Wettability Self-Assembly in Mechanically \\ Induced Self-Cracking
}

Jiazhe $\mathrm{Xu}^{\dagger}, \#$, Zhiguang Qiu ${ }^{\dagger, \#}$, Mingyang Yang ${ }^{\dagger}$, Junwei Chen ${ }^{\dagger}$, Qingyun Luo ${ }^{\dagger}$, Ziyi Wu ${ }^{\dagger}$, Gui-Shi Liu *, Jin $\mathrm{Wu}^{\dagger}$, Zong Qin ${ }^{\dagger}, \mathrm{Bo}-\mathrm{Ru}$ Yang ${ }^{\dagger, *}$

†School of Electronics and Information Technology, State Key Lab of Opto-Electronic Materials \& Technologies, Guangdong Province Key Lab of Display Materials and Technologies, Sun Yat-sen University, Guangzhou 510275, People's Republic of China.

${ }^{\ddagger}$ Guangdong Provincial Key Laboratory of Optical Fiber Sensing and Communication Technology, Department of Optoelectronic Engineering, Jinan University, Guangzhou, 510632, China.

*Email: yangboru@mail.sysu.edu.cn 


$$
\boldsymbol{T}(\%)=\frac{A_{\text {empty }}}{A_{\text {total }}}=\frac{(L-W)^{2}}{L^{2}} \times 100 \%
$$

$T_{\text {triangle }}(\%)=\frac{\frac{\sqrt{3}}{4} g^{2}-6 a g}{\frac{\sqrt{3}}{4} g^{2}-3 a g} \times 100 \%$

$T_{\text {hexagon }}(\%)=\frac{\frac{3 \sqrt{3}}{2} g^{2}-12 a g}{\frac{3 \sqrt{3}}{2} g^{2}-6 a g} \times 100 \%$

$T_{\text {square }}(\%)=\frac{(g-4 a)^{2}}{(g-2 a)^{2}} \times 100 \%$

Where g means the side length of a pattern surrounded by cracks, and a means width of the crack.

$$
\left\{\begin{array}{l}
\sigma_{x x}\left(r_{1}, \theta_{1}\right)=p \sqrt{\frac{a}{2 r_{1}}}\left[\frac{3}{4} \cos \frac{\theta_{1}}{2}+\frac{1}{4} \cos \frac{5 \theta_{1}}{2}\right] \\
\sigma_{x y}\left(r_{1}, \theta_{1}\right)=p \sqrt{\frac{a}{2 r_{1}}\left[\frac{5}{4} \cos \frac{\theta_{1}}{2}-\frac{1}{4} \cos \frac{5 \theta_{1}}{2}\right]} \\
\sigma_{x x}\left(r_{1}, \theta_{1}\right)=p \sqrt{\frac{a}{2 r_{1}}\left[\frac{1}{4} \cos \frac{\theta_{1}}{2}+\frac{1}{4} \cos \frac{5 \theta_{1}}{2}\right]}
\end{array}\right.
$$

Where $\boldsymbol{r}_{\mathbf{1}}, \boldsymbol{\theta}_{\mathbf{1}}$ means parameters of polar coordinates, $p$ means external stress, $a$ means depth of the crack.

The method of line resistance conversion is used to calculate the sheet resistance.

sheet resistance:

$R_{s q}=\frac{\rho}{d}$

line resistance:

$R_{l}=\rho_{w d}^{l}$

so

$R_{s q}=R_{l} \times \frac{w}{l}$

Where $R_{s q}$ means sheet resistance, $R_{l}$ means line resistance, $\rho$ means resistivity, and $w, l$, and $d$ mean length, width, and film thickness of the STE.

$T_{\text {network }}=\frac{T_{S T E}}{T_{\text {substrate }}}$ 
Table S1. Conductive properties with AgNW-based STE with different stretch strategies.

\begin{tabular}{|c|c|c|c|c|c|}
\hline Stretch Strategy & $\begin{array}{l}\text { Initial Resistance } \\
\left(\Omega \cdot \mathrm{sq}^{-1}\right)\end{array}$ & $\begin{array}{l}\text { Transmittance( } \\
\%)\end{array}$ & $\begin{array}{l}\text { Figure of Merit } \\
\text { (FoM) }\end{array}$ & Strain Limit & Reference \\
\hline Direct-Stretching & 15.00 & $93.80 \%$ & 386.43 & $20 \%$ & [3] \\
\hline Direct-Stretching & 42.30 & $85.00 \%$ & 52.64 & $30.00 \%$ & {$[4]$} \\
\hline Wavy Configuration & 26.4 & $85.70 \%$ & 89.01 & $30.00 \%$ & [5] \\
\hline Wavy Configuration & 5.32 & $69.00 \%$ & 173.81 & $60.00 \%$ & [6] \\
\hline Nanomesh & 37 & $77.00 \%$ & 36.49 & $35.00 \%$ & {$[7]$} \\
\hline Welding & 36.20 & $80.00 \%$ & 44.12 & $50.00 \%$ & [8] \\
\hline Welding & 25.00 & $85.00 \%$ & 89.07 & $20.00 \%$ & [9] \\
\hline Welding & 19.50 & $86.80 \%$ & 131.79 & $80.00 \%$ & [10] \\
\hline Alignment & 4.68 & $76.00 \%$ & 273.85 & $10.00 \%$ & [11] \\
\hline Adhesive Encapsulation & 21.60 & $80.00 \%$ & 73.94 & $70.00 \%$ & [12] \\
\hline Adhesive Encapsulation & 30 & $84.00 \%$ & 68.98 & $50.00 \%$ & [13] \\
\hline Adhesive Encapsulation & 10.00 & $82.40 \%$ & 185.47 & $30.00 \%$ & [14] \\
\hline Adhesive Encapsulation & 200 & $75 \%$ & 6.09 & $50 \%$ & {$[15]$} \\
\hline Multi-Layer Lamination & 50 & $75.00 \%$ & 24.37 & $80.00 \%$ & [16] \\
\hline \multicolumn{6}{|l|}{ Hydrophilic } \\
\hline Self-Assembly in & 9.8 & $90.30 \%$ & 360.14 & $125.00 \%$ & Our Work \\
\hline Self-Cracking Crevices & & & & & \\
\hline
\end{tabular}

$\mathbf{F o M}=\frac{\sigma_{D C}}{\sigma_{O p}}=\frac{Z_{0}}{2 R_{s} T^{-1 / 2}-1}$

Where, $\sigma_{D C}$ is the bulk conductivity under DC condition, $\sigma_{O p}$ is the optical conductivity of the material, $Z_{0}$ is the free space impedance (377 $\Omega$ generally), $R_{S}$ is sheet resistance, and $\mathrm{T}$ is transmittance. 

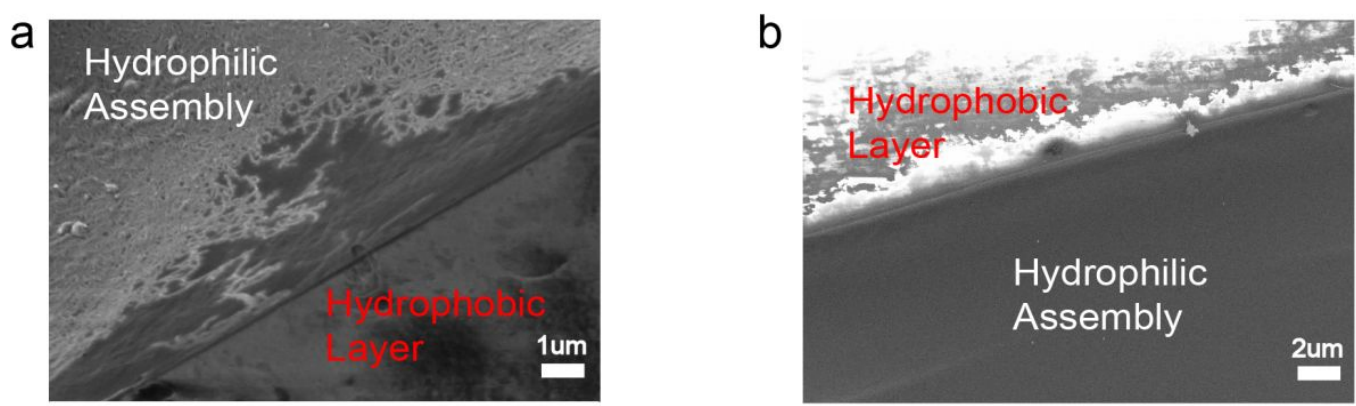

Figure S1. The self-assembly of (a) CNT and (b) PEDOT in hydrophilic channels. 
a

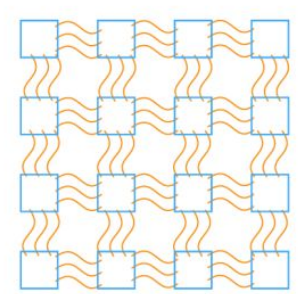

b

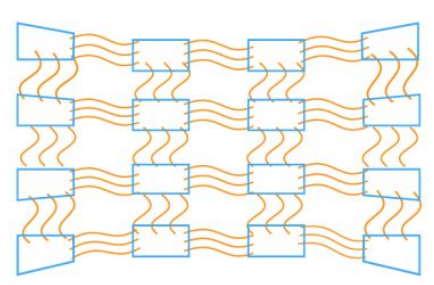

C

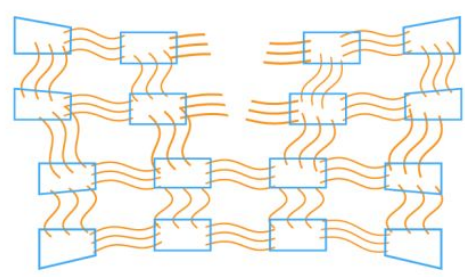

d

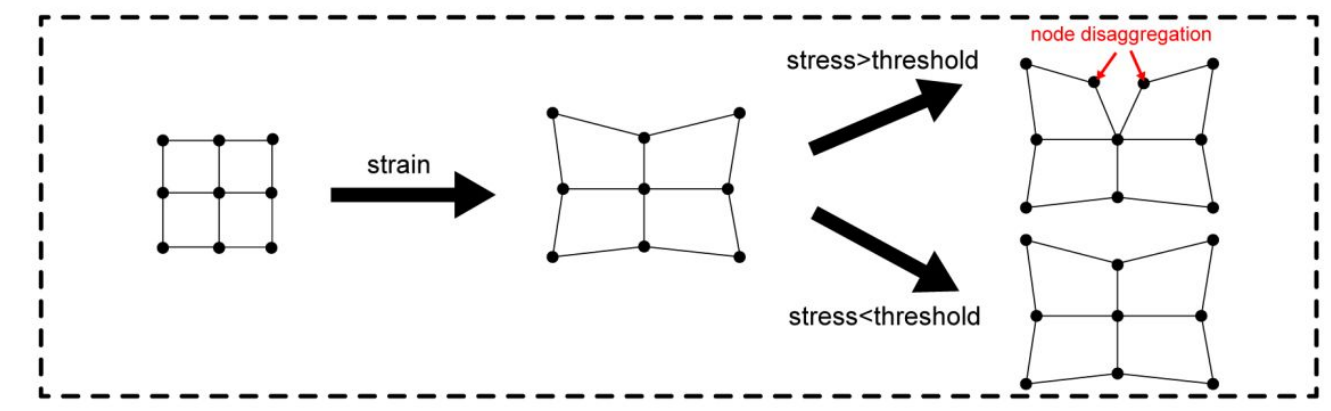

e

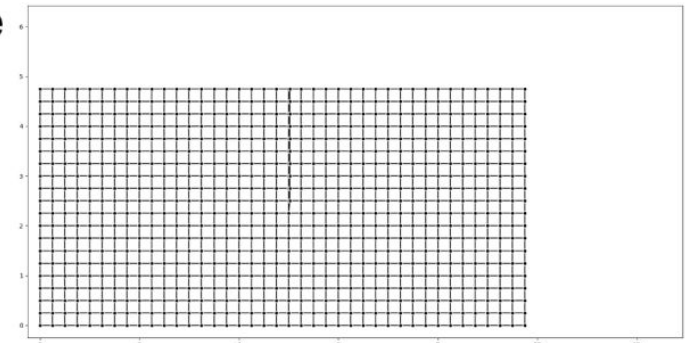

f

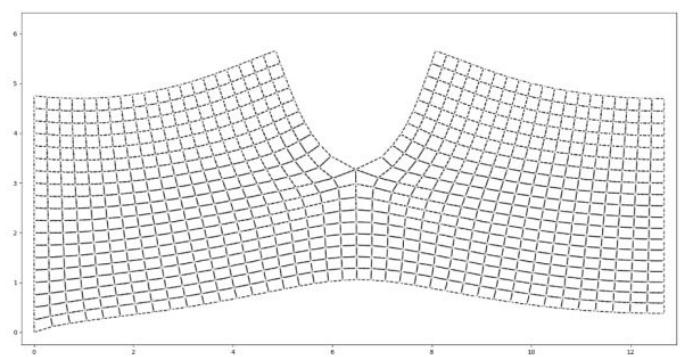

Figure S2. (a-c) RUC model for crack growth. (d) Crack growth detail in RUCFEA. RUCFEs under (e) no strain and (f) $30 \%$ strain. 


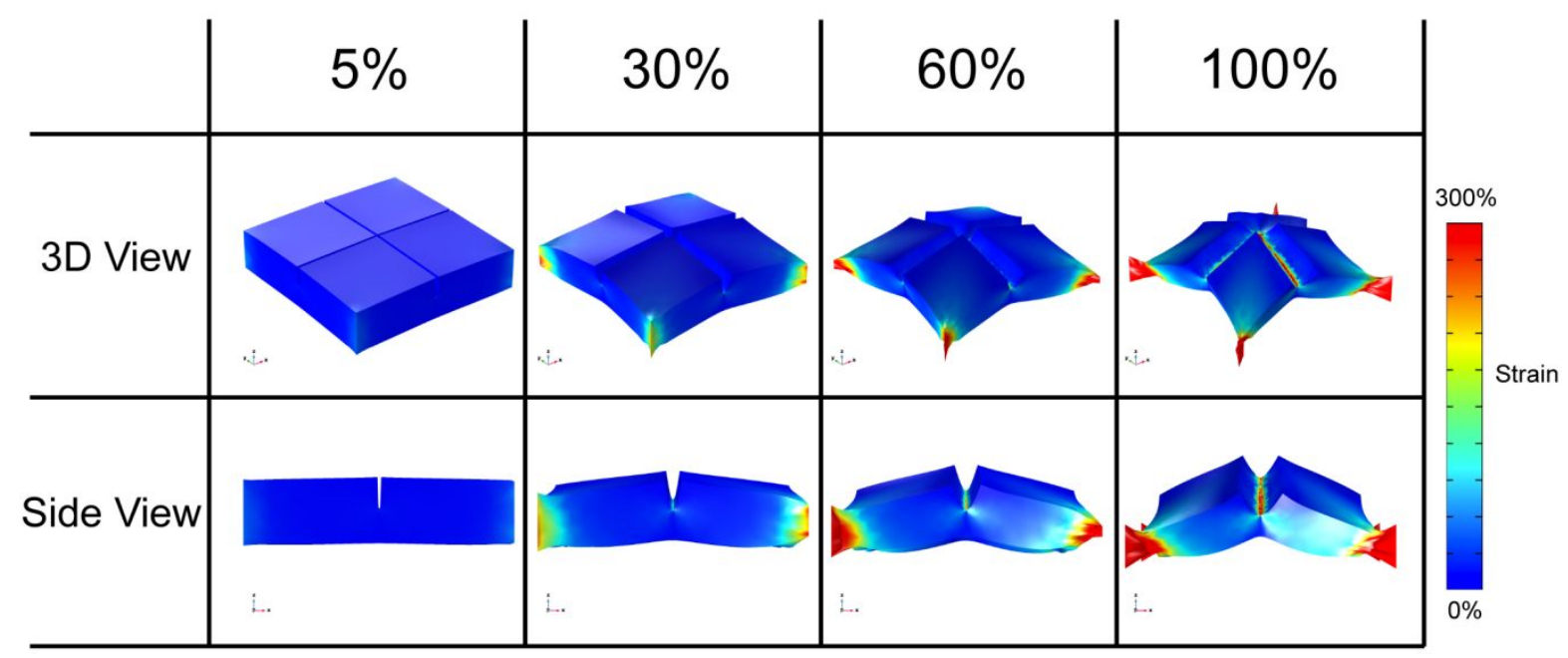

Figure S3. The crack channels deformation under biaxial tension. 


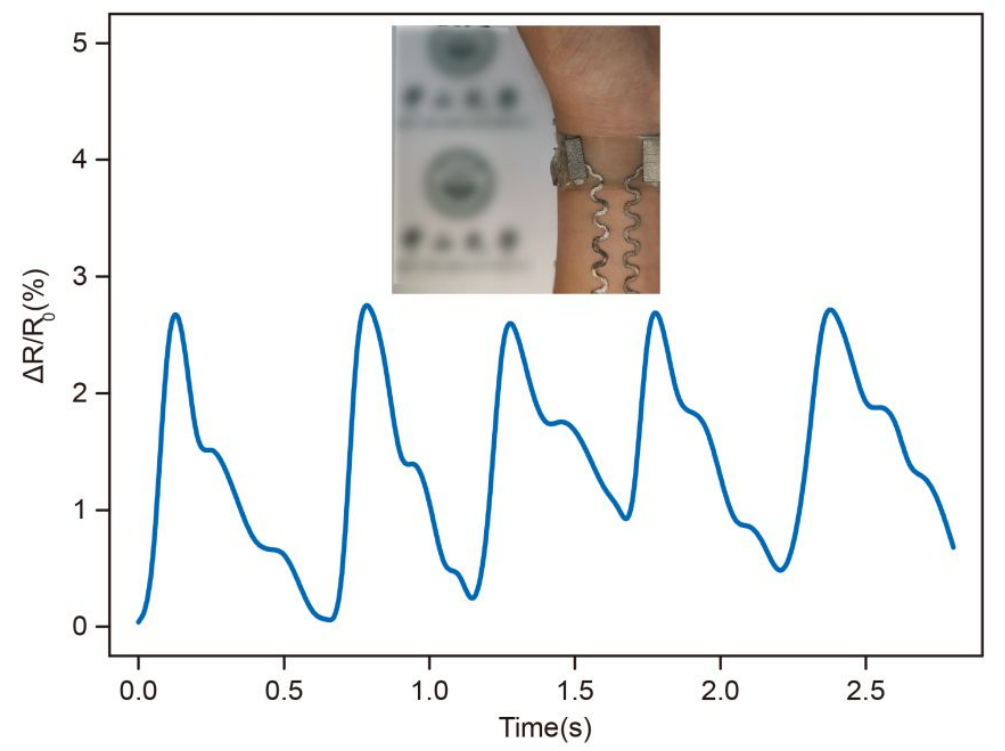

Figure S4. The STE detects pulse wrist gesture. 
a

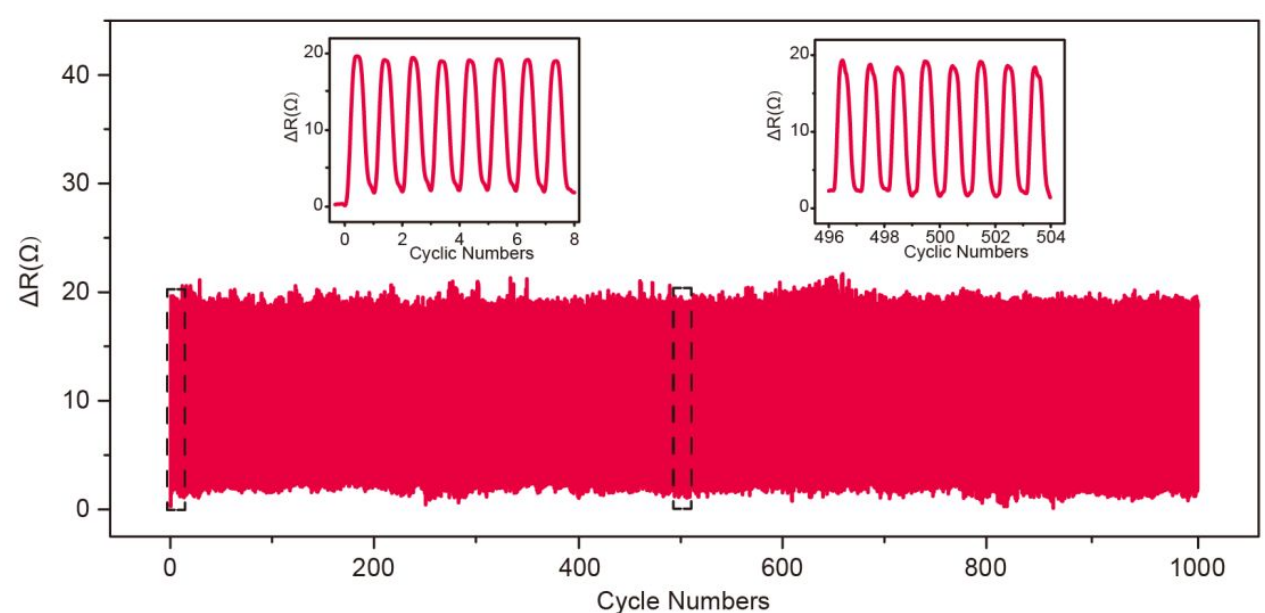

b

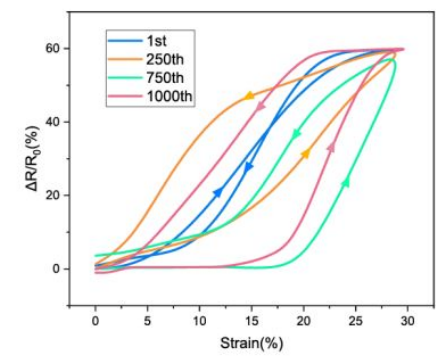

C

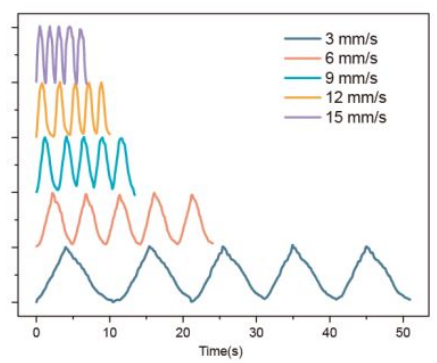

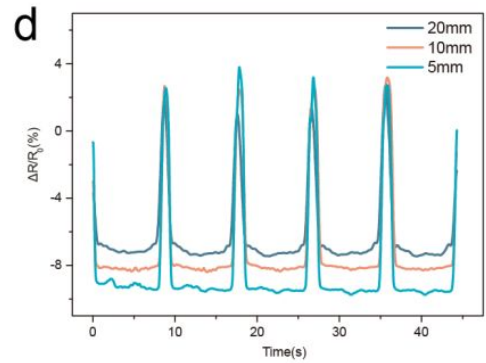

Figure S5. (a) The STE detects $30 \%$ Strain in 0-8 and 496-500 cycles. (b) The STE detects $30 \%$ Strain in the $1^{\text {st }}, 250^{\text {th }}, 750^{\text {th }}$ and $1000^{\text {th }}$ cycles. (c) Detecting $30 \%$ strain with increasing speed. (d) Bending the STE with a radius of 5,10, and $20 \mathrm{~mm}$. 


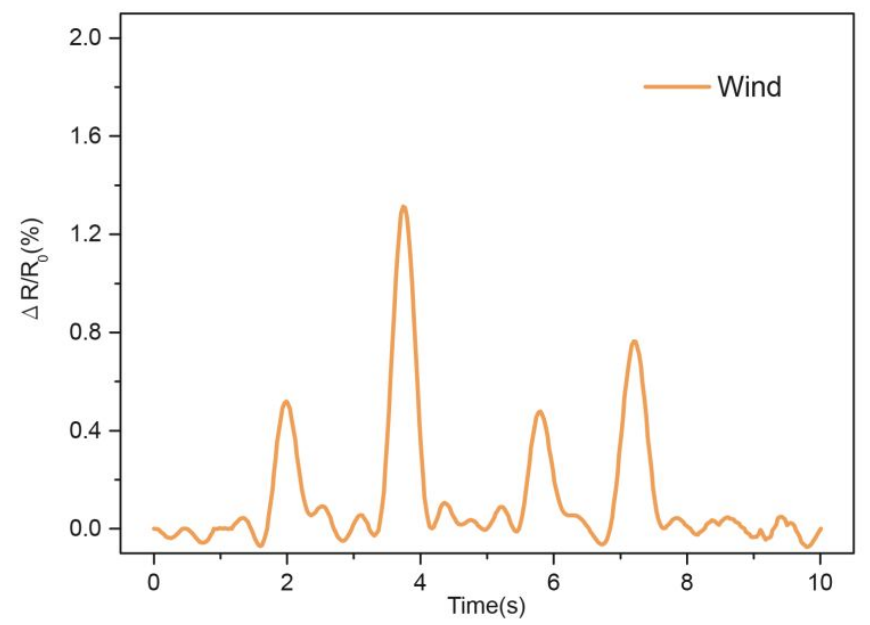

Figure S6. The STE strain sensor detects wind when spread out on the table. 

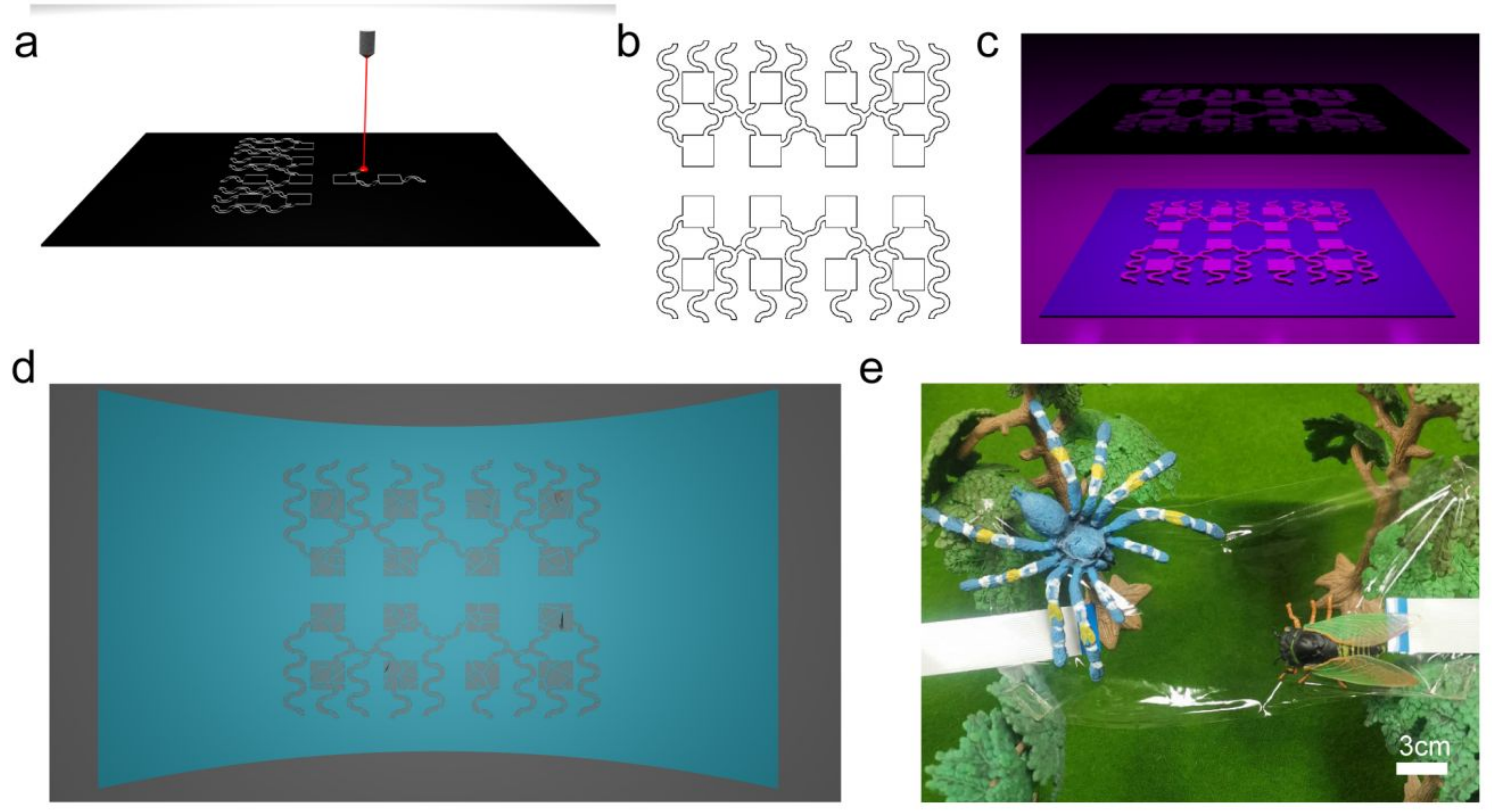

Figure S7. (a) Laser cutting mask. (b) Laser cut the route in CAD. (c) UV-Ozone patterning treatment. (d) Crack patterning growth under strain. (e) A $4 \times 4$ sensing gravity crack-based STE detector array for bio-spider-web. 


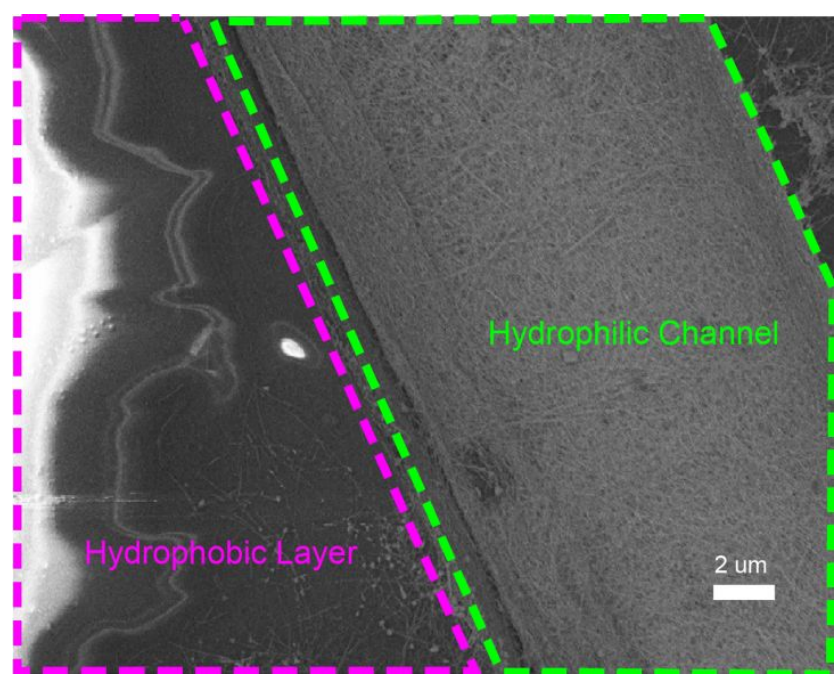

Figure S8. Self-Assembly of AgNWs in Hydrophilic crevice channel on STE. The purple area is the hydrophobic layer where the AgNWs were coated little. The green area is the hydrophilic channel where the AgNWs were coated fully. 


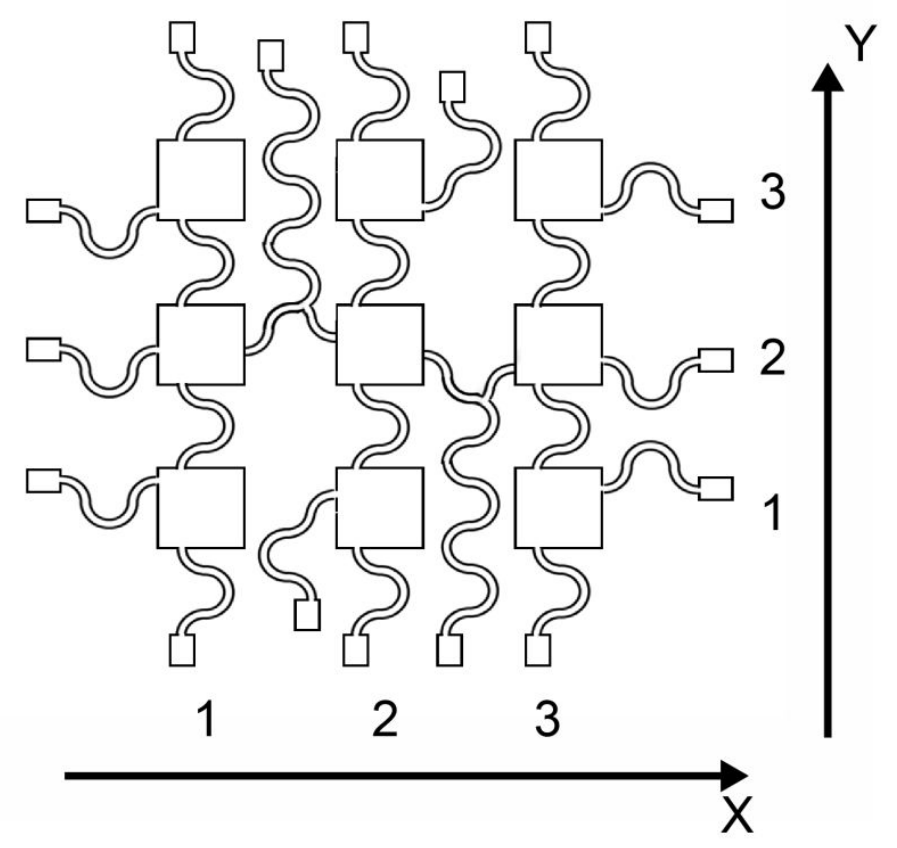

Figure S9. Wire connection of $3 \times 3$ strain sensors in Figure $3 \mathrm{~g}$. 


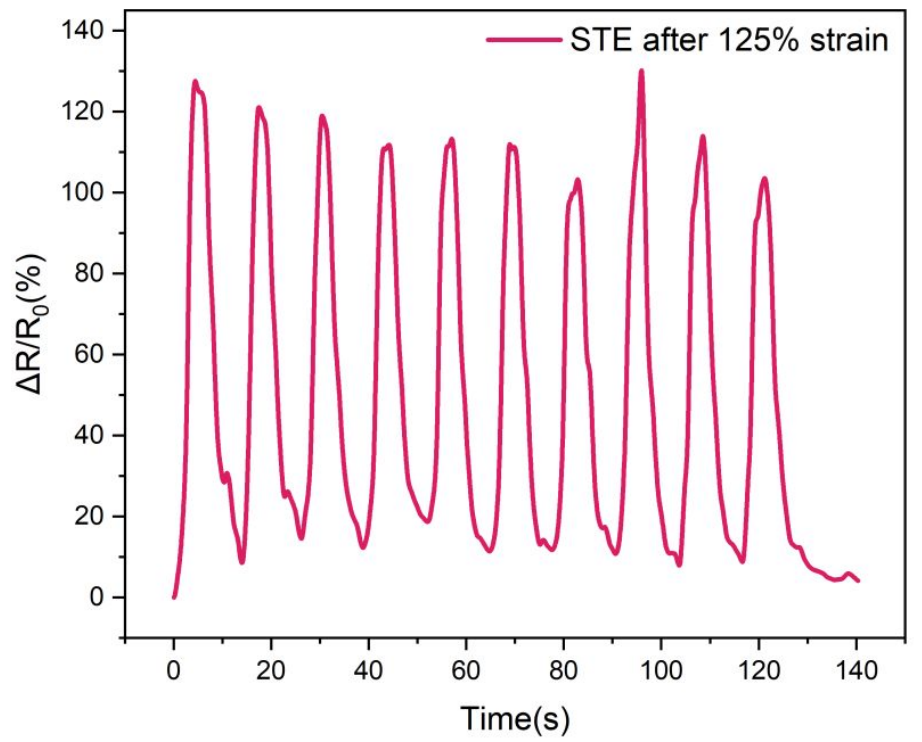

Figure S10. Ten strain/release (30\%) cycles after $125 \%$ strain. 


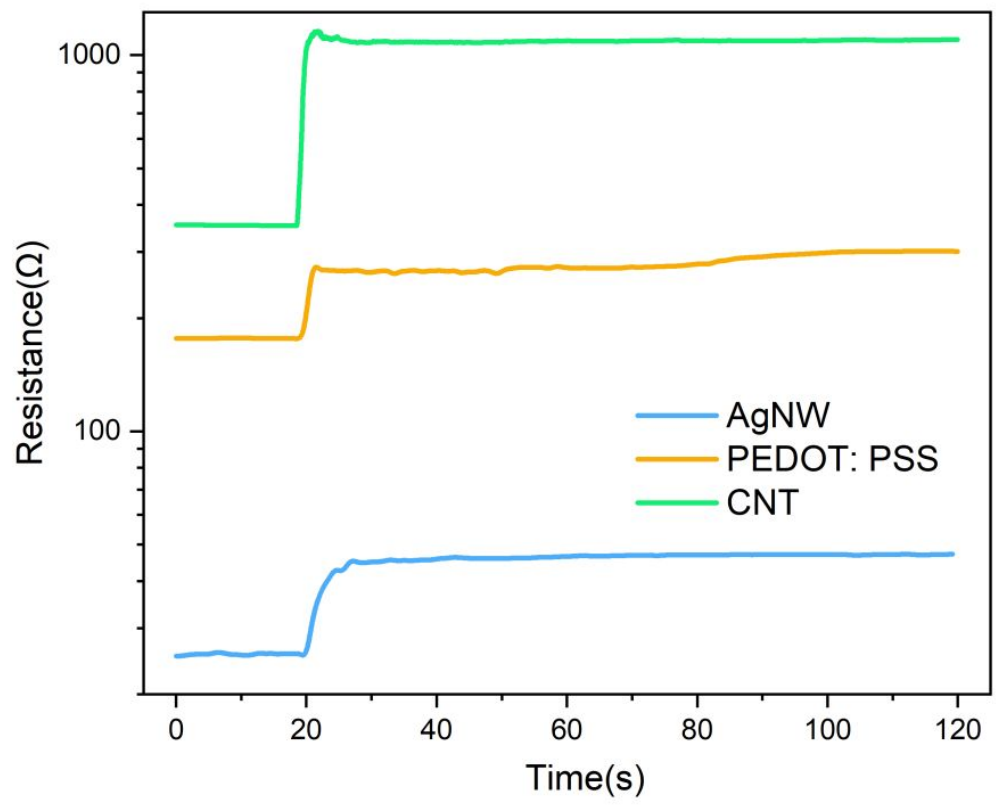

Figure S11. Resistance response to tensile strains (30\% tensile strain) of each strain sensor based on Ag NW, CNT, and PEDOT: PSS with $100 \mathrm{sec}$. 
Table S2. Summary of as-fabricated strain sensor.

\begin{tabular}{lll}
\hline Conductive Materials & Gauge Factor & Reference \\
\hline PEDOT:PSS & $110(30 \%)$ & ${ }^{[18]}$ \\
\hline PEDOT:PSS/AgNW & $12(50 \%)$ & ${ }^{[19]}$ \\
\hline Ionogel & $1.6(200 \%)$ & ${ }^{[20]}$ \\
\hline CNT/MXene & $363(100 \%)$ & ${ }^{[21]}$ \\
\hline $\mathrm{Au}$ & $1000(120 \%)$ & ${ }^{[22]}$ \\
\hline Graphene/AgNF & $1.2 \mathrm{E} 8(400 \%)$ & ${ }^{[23]}$ \\
\hline AgNW & $288(60 \%)$ & This work \\
\hline
\end{tabular}




\section{References}

(1) Li, T.; Li, S.; Li, X.; Xu, Z.; Zhao, J.; Shi, Y.; Wang, Y.; Yu, R.; Liu, X.; Xu, Q. A Leaf Vein-Like Hierarchical Silver Grids Transparent Electrode Towards High-Performance Flexible Electrochromic Smart Windows. Science Bulletin 2020, 65 (3), 225-232.

(2) Sneddon, I. N. The Distribution of Stress in the Neighbourhood of a Crack in an Elastic Solid. Proceedings of the Royal Society of London. Series A. Mathematical and Physical Sciences 1946, 187 (1009), 229-260.

(3) Jung, J.; Lee, H.; Ha, I.; Cho, H.; Kim, K. K.; Kwon, J.; Won, P.; Hong, S.; Ko, S. H. Highly Stretchable and Transparent Electromagnetic Interference Shielding Film Based on Silver Nanowire Percolation Network for Wearable Electronics Applications. ACS applied materials \& interfaces 2017, 9 (51), 44609-44616.

(4) Lee, H.; Hong, S.; Lee, J.; Suh, Y. D.; Kwon, J.; Moon, H.; Kim, H.; Yeo, J.; Ko, S. H. Highly Stretchable and Transparent Supercapacitor by Ag-Au Core-Shell Nanowire Network with High Electrochemical Stability. ACS applied materials \& interfaces 2016, 8 (24), 1544915458 .

(5) Pyo, J. B.; Kim, B. S.; Park, H.; Kim, T. A.; Koo, C. M.; Lee, J.; Son, J. G.; Lee, S.-S.; Park, J. H. Floating Compression of Ag Nanowire Networks for Effective Strain Release of Stretchable Transparent Electrodes. Nanoscale 2015, 7 (39), 16434-16441.

(6) Fan, H.; Li, K.; Li, Q.; Hou, C.; Zhang, Q.; Li, Y.; Jin, W.; Wang, H. PrepolymerizationAssisted Fabrication of an Ultrathin Immobilized Layer to Realize a Semi-Embedded Wrinkled Agnw Network for a Smart Electrothermal Chromatic Display and Actuator. Journal of Materials Chemistry C 2017, 5 (37), 9778-9785.

(7) Cho, J. H.; Ha, S.-H.; Kim, J.-M. Transparent and Stretchable Strain Sensors Based on Metal Nanowire Microgrids for Human Motion Monitoring. Nanotechnology 2018, 29 (15), 155501. (8) Lee, C.; Oh, Y.; Yoon, I. S.; Kim, S. H.; Ju, B.-K.; Hong, J.-M. Flash-Induced Nanowelding of Silver Nanowire Networks for Transparent Stretchable Electrochromic Devices. Scientific reports 2018, 8 (1), 1-10.

(9) Lee, M. S.; Lee, K.; Kim, S. Y.; Lee, H.; Park, J.; Choi, K. H.; Kim, H. K.; Kim, D. G.; Lee, D. Y.; Nam, S.; Park, J. U. High-Performance, Transparent, and Stretchable Electrodes Using Graphene-Metal Nanowire Hybrid Structures. Nano Lett 2013, 13 (6), 2814-21.

(10) Lee, H.-J.; Oh, S.; Cho, K.-Y.; Jeong, W.-L.; Lee, D.-S.; Park, S.-J. Spontaneous and Selective Nanowelding of Silver Nanowires by Electrochemical Ostwald Ripening and High Electrostatic Potential at the Junctions for High-Performance Stretchable Transparent Electrodes. ACS applied materials \& interfaces 2018, 10 (16), 14124-14131.

(11) Ko, Y.; Song, S. K.; Kim, N. H.; Chang, S. T. Highly Transparent and Stretchable Conductors Based on a Directional Arrangement of Silver Nanowires by a Microliter-Scale Solution Process. Langmuir 2016, 32 (1), 366-373.

(12) Kim, J.; Park, J.; Jeong, U.; Park, J. W. Silver Nanowire Network Embedded in Polydimethylsiloxane as Stretchable, Transparent, and Conductive Substrates. Journal of Applied Polymer Science 2016, 133 (33).

(13) Kim, D.-H.; Yu, K.-C.; Kim, Y.; Kim, J.-W. Highly Stretchable and Mechanically Stable Transparent Electrode Based on Composite of Silver Nanowires and Polyurethane-Urea. ACS 
applied materials \& interfaces 2015, 7 (28), 15214-15222.

(14) Jun, S.; Choi, K. W.; Kim, K.-S.; Kim, D. U.; Lee, C.-J.; Han, C. J.; Lee, C.-R.; Ju, B.-K.; Kim, J.-W. Stretchable Photodetector Utilizing the Change in Capacitance Formed in a Composite Film Containing Semiconductor Particles. Composites Science and Technology 2019, 182, 107773.

(16) Fan, X.; Wang, N.; Yan, F.; Wang, J.; Song, W.; Ge, Z. A Transfer-Printed, Stretchable, and Reliable Strain Sensor Using Pedot: Pss/Ag Nw Hybrid Films Embedded into Elastomers. Advanced Materials Technologies 2018, 3 (6), 1800030.

(16) Shin, H.; Sharma, B. K.; Lee, S. W.; Lee, J.-B.; Choi, M.; Hu, L.; Park, C.; Choi, J. H.; Kim, T. W.; Ahn, J.-H. Stretchable Electroluminescent Display Enabled by Graphene-Based Hybrid Electrode. ACS applied materials \& interfaces 2019, 11 (15), 14222-14228.

(17) Kwan, Y. C. G.; Le, Q. L.; Huan, C. H. A. Time to Failure Modeling of Silver Nanowire Transparent Conducting Electrodes and Effects of a Reduced Graphene Oxide over Layer. Solar Energy Materials and Solar Cells 2016, 144, 102-108.

(18) Fan, X.; Wang, N.; Wang, J.; Xu, B.; Yan, F. Highly Sensitive, Durable and Stretchable Plastic Strain Sensors Using Sandwich Structures of Pedot: Pss and an Elastomer. Materials Chemistry Frontiers 2018, 2 (2), 355-361.

(19) Fan, X.; Wang, N.; Yan, F.; Wang, J.; Song, W.; Ge, Z. A Transfer-Printed, Stretchable, and Reliable Strain Sensor Using Pedot: Pss/Ag Nw Hybrid Films Embedded into Elastomers. Advanced Materials Technologies 2018, 3 (6), 1800030.

(20) Sun, J.; Li, R.; Lu, G.; Yuan, Y.; Zhu, X.; Nie, J. A Facile Strategy for Fabricating Multifunctional Ionogel Based Electronic Skin. Journal of Materials Chemistry C 2020, 8 (25), 8368-8373.

(21) Wang, H.; Zhou, R.; Li, D.; Zhang, L.; Ren, G.; Wang, L.; Liu, J.; Wang, D.; Tang, Z.; Lu, G. High-Performance Foam-Shaped Strain Sensor Based on Carbon Nanotubes and Ti3c2t X Mxene for the Monitoring of Human Activities. ACS nano 2021.

(22) Zhu, J.; Wu, X.; Jan, J.; Du, S.; Evans, J.; Arias, A. C. Tuning Strain Sensor Performance Via Programmed Thin-Film Crack Evolution. ACS Appl Mater Inter 2021.

(23) Zhu, L.; Wang, Y.; Mei, D.; Ding, W.; Jiang, C.; Lu, Y. Fully Elastomeric FingerprintShaped Electronic Skin Based on Tunable Patterned Graphene/Silver Nanocomposites. ACS Appl Mater Inter 2020, 12 (28), 31725-31737. 